\title{
Clinicopathological description of 43 oncocytic adrenocortical tumors: importance of Ki-67 in histoprognostic evaluation
}

\author{
Karine Renaudin ${ }^{1}$ - Sarra Smati ${ }^{2}$ - Matthieu Wargny ${ }^{2}$ - Abir Al Ghuzlan ${ }^{3}$ - Sébastien Aubert ${ }^{4}$. \\ Emmanuelle Leteurtre ${ }^{4} \cdot$ Martine Patey $^{5} \cdot$ Mathilde Sibony $^{6} \cdot$ Nathalie Sturm $^{7} \cdot$ Frédérique Tissier $^{8}$. \\ Laurence Amar $^{9}$ - Jérôme Bertherat ${ }^{10}$. Claudine Berthozat ${ }^{11}$. Olivier Chabre ${ }^{11}$. Christine Do Cao ${ }^{12}$. \\ Magalie Haissaguerre ${ }^{13}$. Peggy Pierre ${ }^{14}$. Claire Briet ${ }^{15}$. Delphine Vezzosi ${ }^{16}$. Jean Christophe Lifante ${ }^{17}$. \\ François Pattou $^{18}$ - Eric Mirallie ${ }^{19}$. Eric Baudin ${ }^{20} \cdot$ Bertrand Cariou $^{2} \cdot$ Rossella Libe $^{10,20,21}$. \\ Delphine Drui ${ }^{2}$. for Comete-Cancer Network
}

Received: 16 February 2018 / Revised: 4 April 2018 / Accepted: 8 April 2018 / Published online: 19 June 2018

(c) United States \& Canadian Academy of Pathology 2018

\begin{abstract}
Oncocytic adrenocortical tumors are a rare subtype of adrenal tumors with challenging diagnosis and histoprognostic assessment. It is usually believed that oncocytic adrenocortical tumors have a more indolent clinical behavior than conventional adrenocortical tumors. As the Weiss score overestimates the malignancy of oncocytic adrenocortical tumors owing to intrinsic parameters, alternative scores have been proposed. The Lin-Weiss-Bisceglia score is currently recommended. We performed a large nationwide multicenter retrospective clinicopathologic study of oncocytic adrenocortical tumors. Among the 43 patients in our cohort, 40 patients were alive without disease, 2 patients died of their disease and 1 patient was alive with relapse after a median follow-up of 38 months (20-59). Our data revealed that over 50\% of the oncocytic adrenocortical tumor cases were diagnosed as carcinoma whatever the classification systems used, including the Lin-Weiss-Bisceglia score. The exception is the Helsinki score, which incorporates the Ki-67 proliferation index and was the most specific prognostic score for oncocytic adrenocortical tumor malignancy without showing a loss in sensitivity. A comparison of malignant oncocytic adrenocortical tumors with conventional adrenocortical carcinomas matched for age, sex, ENS@T stage and surgical resection status showed significant better overall survival of malignant oncocytic adrenocortical tumors.
\end{abstract}

\section{Introduction}

Oncocytic adrenocortical tumors are a rare subtype that represent approximately $10 \%$ of adrenocortical tumors [1]. One hundred fifty cases have been reported which were most often single cases or small series with the largest series consisting of 27 cases [1]. By definition, these tumors are made up of at least $50 \%$ oncocytic cells and are mixed $(50-90 \%$ oncocytic cells) or pure $(>90 \%)$. Identification of the oncocytic character of tumor cells is the first diagnostic difficulty. Prognostic evaluation of these tumors is the

These authors contributed equally: Karine Renaudin, Sarra Smati.

Karine Renaudin

karine.renaudin@chu-nantes.fr

Extended author information available on the last page of the article. second difficulty given the multiplicity of scoring systems and the risk of overestimating potential malignancy such as with the Weiss score owing to parameters that are intrinsic to oncocytic cells (eosinophilic character, elevated Fuhrman grade, usually diffuse architectural structure, a minimum Weiss score of 3). The Lin-Weiss-Bisceglia score, specifically developed for this type of tumor [2], has been the most used since publication of the Wong et al. study [3] and was recently recommended by the WHO in 2017 [4]. The reticulin algorithm applicable to all adrenocortical tumors including the oncocytic variant has only been validated by one team $[1,5]$.

The Helsinki score has been very recently proposed as the most relevant prognostic system for all types of adrenocortical tumors [6, 7]. This scoring system associates two Weiss criteria with weighting (necrosis $\times 5$ and mitotic rate $\times 3$ ) and the Ki-67 proliferation index.

When malignancy is confirmed, several elements suggest that oncocytic carcinomas could have a more favorable 
prognosis than conventional adrenocortical carcinomas with a median survival rate that is 2-4 times longer [3].

The present study evaluated, using the National Registry of Adrenocortical Tumors under the aegis of the COMETE Network, the diagnostic and prognostic criteria of oncocytic adrenocortical tumors. We compared the different histoprognostic scores and evaluated TNM/ENS@T stage and the Ki-67 proliferation index. Finally, we compared their prognoses with those of conventional adrenocortical tumors.

\section{Materials and methods}

\section{Case collection}

A search of the pathology files of 15 French institutions (COMETE network) from 2000 to 2014 identified 94 cases of potential oncocytic adrenocortical tumors regardless of ENS@T stage and Weiss or Lin-Weiss-Bisceglia scores. All available hematoxylin and eosin-stained slides, immunohistochemical slides for each case were centrally reviewed by eight pathologists having a specific interest in adrenal pathology (KR, AAG, SA, EL, MP, MS, NS and FT) for validation of their adrenocortical origin and of the oncocytic character of the cell. From this first review, 57 cases were identified as oncocytic adrenocortical tumors showing the expression of at least one of the more reliable markers of the adrenal cortex (MELAN A+, inhibin $\alpha+$ ), no expression of chromogranin A and constituted of at least $50 \%$ oncocytes. Oncocytes were defined morphologically (i.e., granular cytoplasm cells, eosinophilic, round nucleus, voluminous with a nucleolus that is often prominent) and by stronger cytoplasmic immunostaining than normal adrenal cells for anti-mitochondrial antibodies.

Relevant clinical data on the patients were obtained via retrospective review of patient medical files. These data included age, sex, tumor laterality, discovery mode, hormone secretion, tumor size and weight, date and type of surgery (laparoscopy, laparotomy, surgery extended to neighboring organs), extension of the disease on diagnosis, adjuvant treatment (mitotane, radiotherapy, chemotherapy), date of the last follow-up, patient vital status on the date of the last follow-up (alive without relapse, alive with relapse, died from the disease). Follow-up information was updated through June 2016. Fourteen additional cases were excluded owing to a lack of clinical data.

Finally, our study included 43 cases of oncocytic adrenocortical tumors divided into 28 "purely" oncocytic tumors (>90\% oncocytes) and 15 "mixed" tumors (50-90\% oncocytes combined with conventional adrenal tumor cell components).

Histological scores (Lin-Weiss-Bisceglia score [2], Weiss score [8], reticulin algorithm [5] and Helsinki score
[7]), pT staging and determination of ENS@T stage were established during a second review of these 43 selected cases. Reticulin algorithm [5] and the Helsinki score [7] were performed in $38 / 43$ cases when paraffin block was available. The Helsinki score [7] was determined as follows: $(5 \times$ presence of necrosis $)+(3 \times$ mitotic rate $>5$ per 50 high-power fields) + proliferation index value in the most proliferative area of tumor. The thresholds used for malignancy were a total score $\geq 3$ for the Weiss score [8] and a total score $>8.5$ for the Helsinki score [7].

\section{Immunohistochemistry and assessment of proliferation index}

Immunohistochemical staining was performed with mouse monoclonal Ki-67 antibody (clone M7240, Dako, Les Ulis, France) diluted 1:100 with the detection kit envision flex (Dako K8021, Les Ulis, France) in an autostainer Link 48 (Dako, Les Ulis, France). Ki-67 proliferation index of 38 of the 43 cases was evaluated by one pathologist (KR) by manual counting of 2000 tumor cells on camera-captured/ printed image of the area of greatest Ki-67 positivity.

\section{Comparative data collection}

This population was compared with a cohort of 37 patients with conventional adrenocortical carcinomas (Weiss score $\geq 3$ ) followed up in 16 expert referral centers in four European countries (Germany, France, Italy and the Netherlands), consecutively registered in a central computerized database: the European Network for the Study of Adrenal tumors (ENS@T) conventional adrenocortical carcinoma registry. These patients were matched for age, sex, ENS@T stage and surgical resection status. No matching patients were available for six cases. The other data available for these patients were age, sex, hormonal secretion, total Weiss score, adjuvant treatments (mitotane, chemotherapy, radiotherapy), date of the last follow-up, patient vital status on that date.

\section{Statistical analysis}

The main characteristics were presented for all oncocytic adrenocortical tumors and for the subgroups of borderline or malignant tumors following the definition proposed by the different prognostic scores: Lin-Weiss-Bisceglia, Weiss, reticulin algorithm and Helsinki.

For survival analysis, follow-up started on the date of surgery and ended on the date of death, relapse or the most recent information. Survival curves were calculated using the Kaplan-Meier method and presented for all oncocytic adrenocortical tumors and the above-mentioned subgroups with the event of interest being death or relapse. 
Cox multivariate analyses were performed to compare hazard ratios for malignant oncocytic adrenocortical tumors, defined with Helsinki scores and conventional tumors. We adjusted to ENS@T stage and tested the proportional hazards hypothesis. A last analysis was performed after restriction to the only patients treated with mitotane.

A $P$-value $<0.05$ was deemed statistically significant.

All analyses were performed using $\mathrm{R}$ software version 3.3.3 (R Foundation for Statistical Computing, Vienna, Austria) with the survival and survminer packages.

\section{Results}

\section{Clinical features}

Our cohort included 43 patients with oncocytic adrenocortical tumor whose clinical characteristics are presented in Table 1.

Among the 22 functional tumors, 6 produced exclusively cortisol and 8 exclusively androgens, 6 produced both cortisol and androgens and 2 produced estrogen.

As for therapeutic management, all of the patients underwent complete surgical treatment of their tumors (R0). The 14 tumors treated with laparoscopy presented a median size of $4.5 \mathrm{~cm}(3.25-6.3)$ and the 28 treated with laparotomy a median size of $9 \mathrm{~cm}(6.5-13.1)$. Eleven patients underwent surgery extended to neighboring organs. Nineteen patients $(49 \%)$ received adjuvant therapy with mitotane, 4 patients $(11 \%)$ received external radiotherapy ( 2 on the tumor bed and 2 on the metastasis).

The median follow-up of the oncocytic adrenocortical tumor cohort was 38 months (20-59). On the date of the last follow-up, 40 patients were alive without disease, 2 patients had died of their disease and 1 patient was alive with relapse.

In the cohort of 37 conventional adrenocortical carcinomas, all of the patients underwent complete surgical treatment of their tumors (R0), and mitotane treatment was administered to 30 patients $(81 \%)$. The median duration of conventional tumor follow-up was 80 months (40-105). Among the 37 patients with conventional adrenocortical carcinoma, 18 patients presented relapse and among them, 12 died of the disease. The median period between diagnosis and tumor relapse was 10 months (5-23).

\section{Pathology}

The histological characteristics of the 43 oncocytic adrenocortical tumors are presented in Table 2. Most of the oncocytic adrenocortical tumors displayed a typical yellowish-brown color and less often, a pinkish-beige color. The median size was $7.5 \mathrm{~cm}(4.5-10.5)$ and the

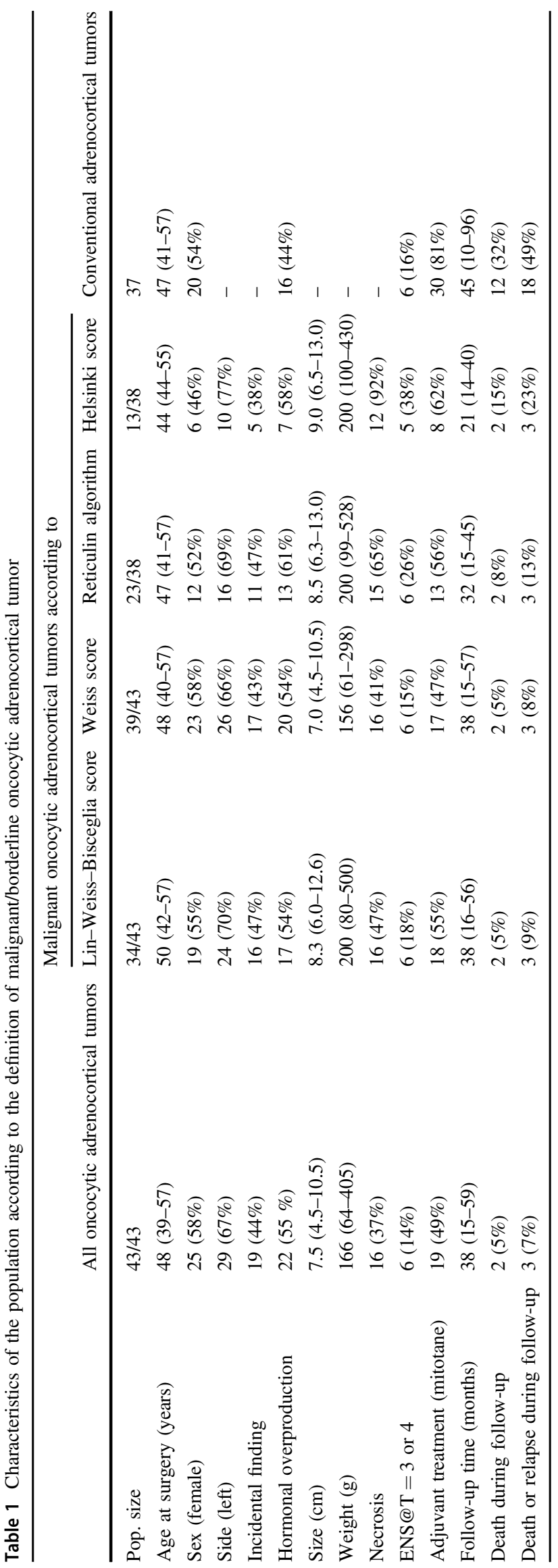


Table 2 Clinical and anatomopathological characteristics of oncocytic adrenocortical tumors, good vs. poor outcomes

\begin{tabular}{|c|c|c|}
\hline Characteristics $^{\mathrm{a}}$ & Good outcome & Poor outcome \\
\hline Sex (female) & $23(57.5 \%)$ & $2 \mathrm{~F} / 1 \mathrm{M}$ \\
\hline Age (years) & $47.5(38.0-55.8)$ & $46,50,59$ \\
\hline Tumor size $(\mathrm{cm})$ & $6.8(4.5-9.9)$ & $8.5,10,21$ \\
\hline Tumor weight (g) & $161(59-392)$ & $80,176,2940$ \\
\hline Hormonal production & $20(54 \%)$ & $\begin{array}{l}\text { 2/3 ( } 1 \text { cortisol, } 1 \\
\text { cortisol and } \\
\text { androgen) }\end{array}$ \\
\hline Pure oncocytic tumors & $26(65 \%)$ & $2 / 3$ \\
\hline Dominant diffuse architecture & $35(88 \%)$ & $2 / 3$ \\
\hline Mitotic index $>5 / 50 \mathrm{HPF}$ & $20(50 \%)$ & $3 / 3$ \\
\hline Atypical mitosis & $21(53 \%)$ & $3 / 3$ \\
\hline Capsular invasion & $9(23 \%)$ & $2 / 3$ \\
\hline Venous invasion & $7(18 \%)$ & $2 / 3$ \\
\hline Sinusoidal invasion & $0(0 \%)$ & $1 / 3$ \\
\hline Necrosis & $13(33 \%)$ & $3 / 3$ \\
\hline Reticulin stain: disrupted & $32(91 \%)$ & $3 / 3$ \\
\hline Ki-67 PI (\%) & $3(1-3.5)$ & $7,14,15$ \\
\hline ENS@T (III or IV) & $\begin{array}{l}3(8 \%) \text { (only } \\
\text { stage III) }\end{array}$ & $\begin{array}{l}3 / 3 \text { III, III and } \\
\text { IV }\end{array}$ \\
\hline \multicolumn{3}{|l|}{ Different approaches by scores } \\
\hline Weiss score & $5(3-6)$ & $7,7,8$ \\
\hline $\begin{array}{l}\text { Lin-Weiss-Bisceglia score } \\
\text { (borderline, malignant) }\end{array}$ & $\begin{array}{l}6 \text { Borderline } \\
(15 \%), 25 \\
\text { Malignant }(63 \%)\end{array}$ & 3 Malignant \\
\hline $\begin{array}{l}\text { Reticulin algorithm } \\
\text { (malignant) }\end{array}$ & $20(56 \%)$ & 3 \\
\hline Helsinki score & $6(3-9.5)$ & $15,22,23$ \\
\hline
\end{tabular}

COMETE study, $N=43$

aFor "Good outcome" tumors, data are described with group size (\%) for categorical data and median (IQR) for quantitative data. For "Poor outcome" tumors, considering the population size $(n=3)$, we listed the exact distribution or the three corresponding values

median weight was $166 \mathrm{~g}$ (64-405). Among the oncocytic adrenocortical tumors, 14 were ENS@T stage I, 23 were ENS@T stage II, 5 were ENS@T stage III including 1 with invasion of the vena cava (pT4NOM0) and 4 with extra-adrenal extension (pT3 including 1 with lymph node metastasis). One tumor was stage IV (pT2NOM1) with an intra-adrenal tumor but with synchronous bone metastasis.

The mean Ki-67 proliferation index was 3.5\% (range: $0-15 \%)$.

The histological data were not itemized for the conventional adrenocortical carcinomas-only their Weiss scores. Their ENS@T stage was identical to their paired oncocytic adrenocortical tumor. Proliferation indexes were not known for the conventional adrenocortical carcinoma cohort.

\section{All oncocytic adrenocortical tumors}

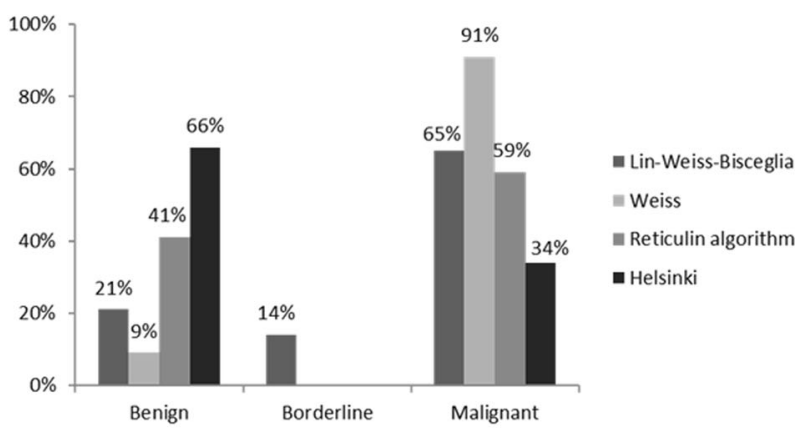

Mixed oncocytic adrenocortical tumors

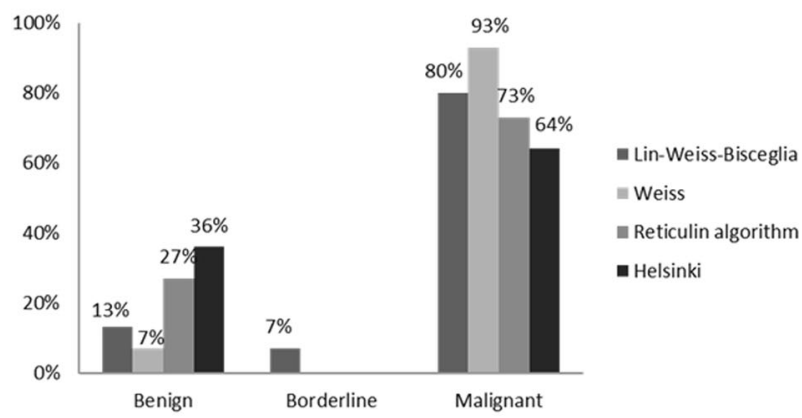

Pure oncocytic adrenocortical tumors

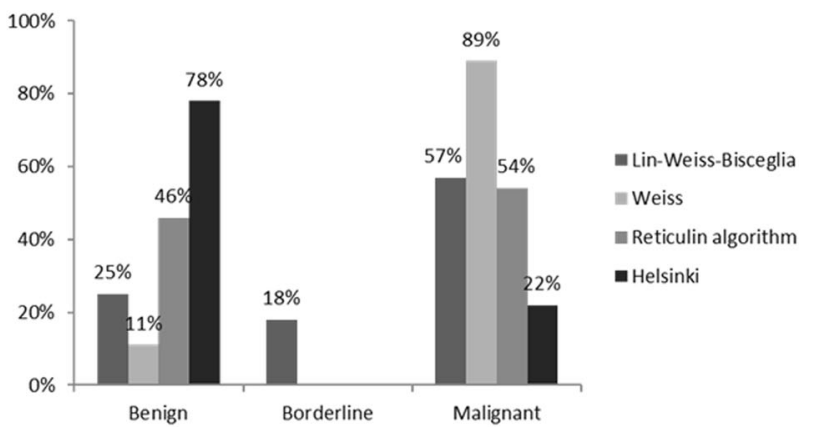

Fig. 1 Classification of our 43 oncocytic adrenocortical tumors according to different histoprognostic scoring systems and to the composition of the tumor (mixed or purely oncocytic)

\section{Oncocytic adrenocortical tumor anatomoclinical correlation}

Among the oncocytic adrenocortical tumor patients, 3 presented an unfavorable outcome with relapse at 2, 3 and 12 months after initial surgery R0. Two died at 23 months. The third patient was still alive with relapse at 24 months postoperatively. The other 40 patients with oncocytic adrenocortical tumor were alive without relapse at the end of follow-up.

Over $50 \%$ of the oncocytic adrenocortical tumors were considered malignant whatever the histoprognostic score used except for the Helsinki score. A breakdown of the oncocytic adrenocortical tumors according to their different scores is presented in Fig. 1 for all of the tumors, then 
Table 3 Number of oncocytic adrenocortical tumor identified as borderline or malignant, concordances between scores

\begin{tabular}{|c|c|c|c|c|}
\hline $\begin{array}{l}\text { Borderline or malignant tumor according to different } \\
\text { prognosis scores }\end{array}$ & Lin-Weiss-Bisceglia score & Weiss score & $\begin{array}{l}\text { Reticulin } \\
\text { algorithm }\end{array}$ & Helsinki score \\
\hline Lin-Weiss-Bisceglia score $(n=34)$ & - & $32 / 34(94 \%)$ & $23 / 30(77 \%)$ & $13 / 30(43 \%)$ \\
\hline Weiss score $(n=39)^{\mathrm{a}}$ & $32 / 39(82 \%)$ & - & $23 / 35(59 \%)$ & $13 / 33(38 \%)$ \\
\hline Reticulin algorithm $(n=23)^{\mathrm{a}}$ & $23 / 23(100 \%)$ & $23 / 23(100 \%)$ & - & $13 / 23(57 \%)$ \\
\hline Helsinki score $(n=13)^{\mathrm{a}}$ & 13/13 (100\%) & $13 / 13(100 \%)$ & $13 / 13(100 \%)$ & - \\
\hline
\end{tabular}

aAlways "malignant" (no definition of borderline)

Fig. 2 Survival curves for death or relapse according to the different definitions of oncocytic adrenocortical tumor malignancy

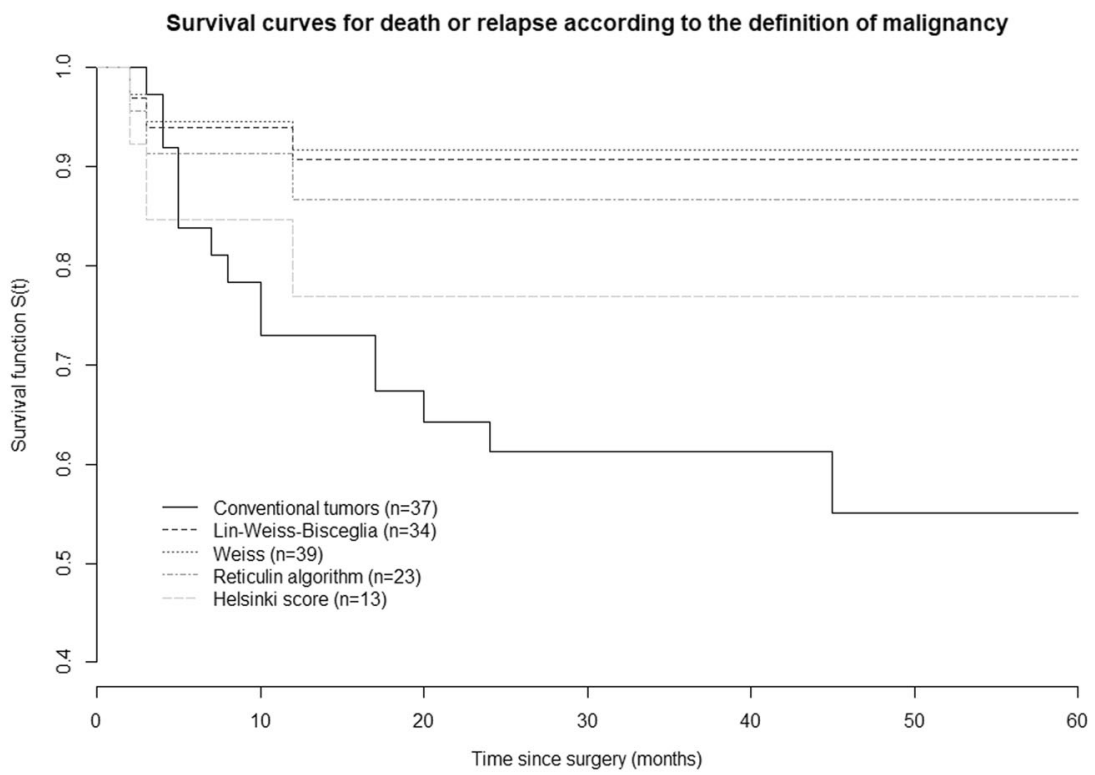

according to their mixed or pure character. Mixed oncocytic tumors were more frequently graded as malignant than the pure oncocytic tumors, whatever the scoring system used.

All of the conventional tumors were malignant according to the Weiss score. The characteristics of the population of malignant oncocytic adrenocortical tumors according to the different scores are compared with the conventional adrenocortical carcinoma characteristics in Table 1.

Table 3 shows the concordance between the four different histoprognostic scores to determine oncocytic adrenocortical tumor malignancy. The Helsinki score was the most discriminating to identify the oncocytic adrenocortical tumors with a propensity for aggressive behavior with a better specificity than the other available scores. It also remained sensitive as all of the tumors with an unfavorable outcome (relapse or death) were graded malignant by the Helsinki score. Finally, all of the tumors graded benign by the Lin-Weiss-Bisceglia and Weiss scores and reticulin algorithm were also graded benign by the Helsinki score.

Discordance was observed in six cases between the Lin-Weiss-Bisceglia score and the other scores. These discordances were major (malignant with Lin-Weiss-Bisceglia score vs. benign with reticulin algorithm and Helsinki score) in three cases owing to the presence of atypical mitoses isolated as the only major criterion of malignancy according to the Lin-Weiss-Bisceglia score. These discordances were minor (borderline with Lin-Weiss-Bisceglia score vs. benign with all the other scores) in three other cases owing to tumor weight $>200 \mathrm{~g}$ and/or tumor size $>10 \mathrm{~cm}$ as the only minor criterion of malignancy.

A comparison of patient pathological and clinical characteristics according to their outcome is presented in Table 2. The tumors with an unfavorable outcome had a higher Weiss score than those with a favorable outcome. The only discriminating morphological criterion between the tumors with a favorable outcome and those with an unfavorable outcome appeared to be the presence of necrosis. The median Ki-67 proliferation index for good prognosis oncocytic adrenocortical tumors was 3\% (1-3) for values at 7, 14 and $15 \%$ for the three tumors with an unfavorable outcome.

\section{Survival analyses}

Survival in patients with oncocytic adrenocortical tumor was significantly higher than in patients with conventional adrenocortical carcinoma (Fig. 2), with survival at 2 years equal to $92 \%$ (95\% confidence interval, $84-100 \%)$ vs. $61 \%(95 \%$ confidence interval, 47-80\%), respectively, $P=0.00074$. We 
Table 4 Hazard ratios associated with patient characteristics, using the Helsinki score to define malignant oncocytic adrenocortical tumors

\begin{tabular}{llll}
\hline & Hazard ratio & $95 \%$ confidence interval & $P$-value \\
\hline Model A ( $N=50,21$ events) & & & \\
Tumor type (oncocytic/conventional) & 0.45 & {$[0.13 ; 1.59]$} & 0.21 \\
ENS@T (3-4 vs. 1-2) & $\mathbf{3 . 2 3}$ & {$[\mathbf{1 . 3 2} ; \mathbf{7 . 9 1}]$} & $\mathbf{0 . 0 1 0}$ \\
Model B $(N=38,21$ events) & & & 0.41 \\
Tumor type (oncocytic/conventional) & 0.58 & {$[0.16 ; 2.09]$} & 0.10 \\
ENS@T (3-4 vs. 1-2) & 2.16 & {$[0.86 ; 5.39]$} & \\
\hline
\end{tabular}

Model A: malignant oncocytic adrenocortical tumors according to Helsinki score + conventional tumors, treated or not by mitotane

Model B: malignant oncocytic adrenocortical tumors according to Helsinki score + conventional tumors, treated by mitotane

Results with $P$-value $<0.05$ are marked in bold compared the outcomes of oncocytic adrenocortical tumors graded malignant or borderline with those of conventional adrenocortical carcinomas. Whatever the scoring system used, malignant or borderline oncocytic adrenocortical tumors outcomes were better than those of conventional adrenocortical carcinomas. However, the Lin-Weiss-Bisceglia and Weiss scores appeared to grossly overestimate oncocytic adrenocortical tumor malignancy potential, whereas reticulin algorithm and more especially the Helsinki score appeared to provide the closest prognoses to those of conventional carcinomas. When oncocytic adrenocortical tumors were malignant according to the Helsinki score and were associated with the conventional adrenocortical carcinoma, the risk of death or relapse after surgery was significantly greater for patients with ENS@T > II (hazard ratio=3.23, 95\% confidence interval [1.32-7.91]). Malignant oncocytic adrenocortical tumors appeared to have a better prognosis than conventional adrenocortical carcinomas but the corresponding hazard ratio did not reach statistical significance (hazard ratio $=0.45$, confidence interval [0.13-1.59]). The same tendencies were found after exclusion of the patients not treated by mitotane (Table 4).

\section{Discussion}

This retrospective multicenter study analyzed the pathological and clinical data of 43 patients with oncocytic adrenocortical tumor and compared their outcomes with similar patients with conventional adrenocortical carcinoma. To our knowledge, this is the largest reported series of oncocytic adrenocortical tumors.

Diagnosis of oncocytic adrenocortical tumor presents two principal problems: on one hand, determination of the oncocytic character of the tumor cells and on the other hand, evaluation of their prognosis.

Determination of oncocytic character is based on subjective morphological criteria. Indeed, among the 94 tumors reviewed by the pathologists, 37 were rejected because the cells were not consensually considered as oncocytic or because there was too small a proportion of oncocytic cells $(<50 \%)$. Anti-mitochondrial immunostaining can help confirm a diagnosis [3] but its quantitative evaluation compared with normal adrenal tissue also remains quite subjective. Such diagnostic subjectivity is a major limitation to the use of a histoprognostic score that is specific to this variant such as the Lin-Weiss-Bisceglia score. It would therefore be preferable to be able to evaluate the prognosis of these tumors independently of their histologic variant. When confronted with an oncocytic tumor, it is essential to confirm its adrenocortical nature before any classification because certain cases can be misdiagnosed as pheochromocytoma [9].

The clinical characteristics of our oncocytic adrenocortical tumor population are superimposable with those described in the two largest published series [1-3] with patients of similar ages, a predominance of women and preferential involvement of the left adrenal gland.

In the present study, the median size and the weight of oncocytic adrenocortical tumors were greater than those usually described for conventional adrenocortical tumors and were similar to the aforementioned series [1-3]. We did not observe a notable difference in weight or size between benign and malignant oncocytic adrenocortical tumors patients, which confirmed that in this tumor variant, these morphometric data are not predictive of biological behavior. Contrary to what has been reported for conventional tumors [10], the proportions of secreting tumors were very close for both benign and malignant oncocytic adrenocortical tumor patients, respectively, 54 and $66 \%$. In agreement with the literature, the most frequent secretions were androgens, then cortisol and much more rarely, estrogen [3].

The histological scores currently applied to oncocytic adrenocortical tumors do not seem to correlate with the risk of recurrence or metastasis. The Lin-Weiss-Bisceglia score, Weiss score and reticulin algorithm ranked tumors, respectively, 28, 39 and 23 as carcinomas, whereas only 3 cases of these series evolved unfavorably with a median follow-up of 38 months. 
It is well recognized that the Weiss score is not suitable for this type of tumor [11] owing to its constitution $(\leq 25 \%$ clear cells, diffuse architecture, high nuclear grade). This was confirmed in this study with 39 out of 43 tumors considered as malignant despite an overall good prognosis.

Alternative scores have been proposed. The most recognized is the Lin-Weiss-Bisceglia score [2]. This score no longer takes into account histologic criteria that are more subjective or unsuited in histologic variants. However, in our study, the Lin-Weiss-Bisceglia score also appeared to overestimate the number of malignant tumors in a number of cases ( 6 borderline and 28 malignant in 43 tumors) and presented diagnosis discrepancies with the other scores especially when there were isolated criteria such as atypical mitosis or major weight or size as unique malignancy criteria. This high level of malignancy diagnosis based on Lin-Weiss-Bisceglia criteria differs from the meta-analysis in the literature, which had validated this score (22\% malignant tumors among 110 cases) [3]. However, these 110 cases were obtained after analyses from the literature, which did not enable central reviewing in order to standardize the tumors. Moreover, in the same publication, the authors reported results that were similar to ours with $61 \%$ malignant tumors according to Lin-Weiss-Bisceglia score in their own cohort of 13 patients with purely oncocytic adrenocortical tumors. In the study of 27 oncocytic adrenocortical tumors reported by Duregon et al. [1], $40 \%$ of the purely oncocytic adrenocortical tumors were classified as malignant according to the Lin-Weiss-Bisceglia score, but this score was not applied to mixed oncocytic adrenocortical tumors, which were classified according to the Weiss score. The high percentage of malignancy that we observed with the Lin-Weiss-Bisceglia score can perhaps be explained by the fact that we used purely oncocytic adrenocortical tumors, as well as mixed oncocytic adrenocortical tumors (oncocyte component between 50 and $90 \%$ of tumor cells). Indeed, most authors suppose that the prognosis with mixed tumors is less favorable than that with purely oncocytic adrenocortical tumors even if there are no data proving the contrary $[1,3]$. In our study, "mixed" oncocytic tumors were certainly graded as malignant more frequently than "pure" oncocytic tumors but two out of the three patients who presented an unfavorable outcome presented purely oncocytic adrenocortical tumors.

Reticulin algorithm is another alternative proposed for the prognosis of oncocytic adrenocortical tumors [1]. Twentythree out of 38 tumors were classified as malignant. No tumor classified as benign with the reticulin algorithm had a poor outcome. However, both quantitative and qualitative interpretations in the reticulin framework remain subjective with a heterogenous pattern especially with the oncocytic variant, which requires analysis of several different samples for optimal interpretation [1]. In our series, $91 \%$ of the tumors with a benign outcome presented a disrupted reticulin structure that is a poorly discriminating element, which finally leads to classification of the tumors essentially according to the three other algorithm criteria, which are mitotic index $>5 / 50$ high-power fields, necrosis and venous invasion or the morphologic items in the Helsinki score.

The Helsinki score appeared to be more specific in our cohort in that it only graded 13 tumors as malignant. This score was recently developed for conventional adrenocortical carcinomas [7] and was very recently validated for adrenocortical carcinoma variants, in particular, the oncocytic variant [6]. In addition to morphological criteria, it uses the Ki-67 proliferation index, which is recognized as an independent prognostic element in localized conventional adrenocortical carcinomas [12]. The morphological criteria used, necrosis and mitotic index, are among the most reproducible Weiss score criteria [13] and the best correlated to conventional adrenocortical carcinomas prognosis [5, 14]. It no longer takes into account histologic criteria that are more subjective or unsuited in histologic variants.

The Ki-67 proliferation index also appears to be of prognostic value in cases of oncocytic variants. In the originator article by Bisceglia [2], Ki-67 was correlated with the mitotic index and was $\geq 5 \%$ for tumors graded malignant and $\leq 4 \%$ for borderline or benign tumors. In their review, Ohtake et al. [15] reported that a Ki-67>10\% was correlated with the presence of significant histologic malignancy signs such as venous invasion, invasion of neighboring organs, or metastasis. In our series, the vast majority of tumors with a favorable outcome had a Ki-67 $\leq 5 \%(n=31$ out of 39) and none had a Ki-67 $>10 \%$. Among the three tumors that had a poor outcome, the two patients who died had a Ki-67 > 10\% and one patient with a known relapse had a $\mathrm{Ki}-67=7 \%$. Ki67 can therefore provide interesting information on the prognosis of oncocytic adrenocortical tumors.

Moreover, in our study necrosis was the only histological criteria that was significantly different between oncocytic tumors with a poor or good outcome whereas it is one of the two histological criteria of the Helsinki score [7].

The Helsinki score has been shown to outperform the most established prognostic parameters for conventional adrenocortical carcinomas such as clinical stage, mitotic index and the Ki-67 proliferation index alone [6] and appeared to be relevant in our oncocytic adrenocortical tumors series mostly with the recently proposed new cutoffs of $<13$ and $\geq 19$ [6].

Initially, the cutoff point for the Helsinki score to identify conventional adrenocortical carcinoma was 8.5 with excellent sensitivity and specificity (100\% and 99.4\%) [7]. In a larger series of tumors that not only included conventional adrenocortical carcinomas but also myxoid and oncocytic variants, Duregon et al. determined new cutoffs of $<13$ and $\geq 19$ for the Helsinki score, enabling better stratification of the tumors in the three prognostic groups [6]. In our series, 
among the 13 tumors graded malignant according to the Helsinki score (Helsinki score > 8.5), 5 had a score $\geq 13$ including 3 tumors with a poor outcome and only the two patients who died had a score $\geq 19$. Our results therefore validate the prognostic value of the Helsinki score with the new cutoffs ( $<13$ and $\geq 19$ ) proposed by Duregon et al [6] for the clinical management of oncocytic adrenocortical tumors.

All of the patients in the cohort were treated in specialized surgical centers with an initial R0. Only 3 of the 43 tumors relapsed. They had initial histological scores that classified the tumors as malignant with good overall sensitivity. Ki-67 was high for the three cases and an ENS@T stage $\geq$ III on diagnosis was pejorative. For the three patients, relapse occurred during the first year of monitoring. This limited number of cases is obviously not enough to draw conclusions but the tumors with poor outcomes were initially aggressive with an early relapse time. Compared with conventional adrenal tumors known to be aggressive with a median overall survival of 32 months and a 5-year survival rate of $38.6 \%$ [16], there is some evidence that the prognosis of malignant oncocytic adrenocortical tumors, at least the pure form, is more favorable (58 months vs. 32 months median survival) [3] but this has yet to be confirmed by a dedicated prospective study.

In the present study, comparison of the malignant oncocytic adrenocortical tumors with the conventional adrenocortical carcinomas showed that the malignant oncocytic adrenocortical tumors had a better prognosis than the conventional tumors even by only retaining the oncocytic adrenocortical tumors graded malignant by the most specific prognostic score (Helsinki score). We then analyzed survival in the only patients treated with mitotane. This subgroup enabled homogenization of the population (patients with conventional and oncocytic tumors) for the initial prognosis because the patients treated with mitotane were considered as the most "severe" by the clinician. Analysis of this subgroup also enabled homogenization in the follow-up of patients treated with mitotane since they generally required closer monitoring. Survival of the patients with malignant oncocytic adrenocortical tumors treated with mitotane remained better than that of the conventional adrenocortical carcinoma patients with the same treatment.

The main limitation of this study is its retrospective nature and therefore a lack of completeness of reported cases. Nevertheless, the study was based on the national pathological registry (COMETE network), which provided broad screening. We did not have access to the clinical data of 14 patients. These patients were not treated in referring centers, making access to medical files impossible. Finally, we unfortunately did not have the Ki-67 for most of the conventional adrenocortical carcinoma patients because when these tumors were analyzed, determination of the cell proliferation score by Ki-67 was not routinely performed.
However, it would be interesting to continue this work by comparing the outcomes of oncocytic adrenocortical tumors and conventional adrenocortical carcinoma patients with the same Helsinki scores.

\section{Conclusion}

This cohort is the largest reported series of oncocytic adrenocortical tumors. This would appear to be in favor of a more indolent outcome of malignant oncocytic adrenocortical tumors in comparison with conventional adrenocortical carcinomas with a limited number of cases with poor outcomes. The histological scores currently and widely used seem to overestimate the potential of malignancy of these tumors except for the Helsinki score, which incorporates the $\mathrm{Ki}-67$ proliferation index and is the most specific prognostic score without showing loss in sensitivity. However, even with a definition of a malignant oncocytic adrenocortical tumor based on the Helsinki score, our results still point to a better prognosis than for conventional tumors.

Acknowledgements For their contributions to this work, we thank: Peter Tucker, Marie-Laure Quintyn-Ranty, Myriam Decaussin, Serge Guyetant, Hélène Trouette, Laurent Doucet, Tchao Meatchi, Camille Buffet, Emmanuel Sonnet, Bénédicte Decoudier, Frédérique Toulgoat, Cécile Caillard, Catherine Ansquer and Maëlle Le Bras.

\section{Compliance with ethical standards}

Conflict of interest The authors declare that they have no conflict of interest.

\section{References}

1. Duregon E, Volante M, Cappia S, et al. Oncocytic adrenocortical tumors: diagnostic algorithm and mitochondrial DNA profile in 27 cases. Am J Surg Pathol. 2011;35:1882-93.

2. Bisceglia M, Ludovico O, Di Mattia A, et al. Adrenocortical oncocytic tumors: report of 10 cases and review of the literature. Int J Surg Pathol. 2004;12:231-43.

3. Wong DD, Spagnolo DV, Bisceglia M, et al. Oncocytic adrenocortical neoplasms-a clinicopathologic study of 13 new cases emphasizing the importance of their recognition. Hum Pathol. 2011;42:489-99.

4. Giordano TJ, Chrousos GP, de Krijger RR, et al. Adrenal cortical carcinoma. In: Lloyd RV, Osamura RY, Klöppel G, Rosai J, editors. The WHO Classification of Tumours of Endocrine Organs. 4th edn. Lyon, PA: International Agency for Research on Cancer, 2017. p. 163-8.

5. Volante M, Bollito E, Sperone P, et al. Clinicopathological study of a series of 92 adrenocortical carcinomas: from a proposal of simplified diagnostic algorithm to prognostic stratification. Histopathology. 2009;55:535-43.

6. Duregon E, Cappellesso R, Maffeis V, et al. Validation of the prognostic role of the "Helsinki Score" in 225 cases of adrenocortical carcinoma. Hum Pathol. 2017;62:1-7. 
7. Pennanen M, Heiskanen I, Sane T, et al. Helsinki score-a novel model for prediction of metastases in adrenocortical carcinomas. Hum Pathol. 2015;46:404-10.

8. Weiss LM, Medeiros LJ, Vickery AL Jr. Pathological features of prognostic significance in adrenocortical carcinoma. Am J Surg Pathol. 1989;13:202-6.

9. Duregon E, Volante M, Bollito E, et al. Pitfalls in the diagnosis of adrenocortical tumors: a lesson from 300 consultation cases. Hum Pathol. 2015;46:1799-807.

10. Allolio B, Fassnacht M. Clinical review: adrenocortical carcinoma: clinical update. J Clin Endocrinol Metab. 2006;91:2027-37.

11. Lin BT, Bonsib SM, Mierau GW, et al. Oncocytic adrenocortical neoplasms: a report of seven cases and review of the literature. Am J Surg Pathol. 1998;22:603-14.
12. Beuschlein F, Weigel J, Saeger W, et al. Major prognostic role of Ki-67 in localized adrenocortical carcinoma after complete resection. J Clin Endocrinol Metab. 2015;100:841-9.

13. Tissier F, Aubert S, Leteurtre E, et al. Adrenocortical tumors: improving the practice of the Weiss system through virtual microscopy: a National Program of the French Network INCaCOMETE. Am J Surg Pathol. 2012;36:1194-201.

14. Assié G, Antoni G, Tissier F, et al. Prognostic parameters of metastatic adrenocortical carcinoma. J Clin Endocrinol Metab. 2007;92:148-54.

15. Ohtake H, Kawamura H, Matsuzaki M, et al. Oncocytic adrenocortical carcinoma. Ann Diagn Pathol. 2010;14:204-8.

16. Bilimoria KY, Shen WT, Elaraj D, et al. Adrenocortical carcinoma in the United States: treatment utilization and prognostic factors. Cancer. 2008;113:3130-6.

\section{Affiliations}

Karine Renaudin ${ }^{1} \cdot$ Sarra Smati ${ }^{2} \cdot$ Matthieu Wargny ${ }^{2} \cdot$ Abir Al Ghuzlan $^{3} \cdot$ Sébastien Aubert $^{4}$.

Emmanuelle Leteurtre ${ }^{4}$ Martine Patey ${ }^{5} \cdot$ Mathilde Sibony $^{6} \cdot$ Nathalie Sturm $^{7} \cdot$ Frédérique Tissier $^{8}$. Laurence Amar ${ }^{9}$. Jérôme Bertherat ${ }^{10}$. Claudine Berthozat ${ }^{11}$. Olivier Chabre ${ }^{11}$. Christine Do Cao ${ }^{12}$.

Magalie Haissaguerre ${ }^{13}$. Peggy Pierre ${ }^{14}$. Claire Briet ${ }^{15}$. Delphine Vezzosi ${ }^{16}$. Jean Christophe Lifante ${ }^{17}$.

François Pattou ${ }^{18}$ - Eric Mirallie ${ }^{19} \cdot$ Eric Baudin $^{20} \cdot$ Bertrand Cariou $^{2} \cdot$ Rossella Libe $^{10,20,21}$.

Delphine Drui' ${ }^{2}$ for Comete-Cancer Network

1 Department of Pathology, CHU Nantes, 44093 Nantes, France

2 Department of Endocrinology, Diabetes and Nutrition, L'institut du Thorax, CHU Nantes, 44093 Nantes, France

3 Department of Pathology, Gustave Roussy Cancer Center, 94805 Villejuif, France

4 Institute of Pathology, CHRU Lille, 59037 Lille, France

5 Department of Pathology, CHU Reims, 51092 Reims, France

6 Department of Pathology, Hôpital Cochin, 75014 Paris, France

7 Department of Pathology, CHU Grenoble-Alpes, 38043 Grenoble, France

8 Department of Pathology, Hôpital Pitié Salpétrière - Université Pierre et Marie Curie, 75013 Paris, France

9 Hypertension Unit, Hôpital Européen Georges Pompidou, 75015 Paris, France

10 Department of Endocrinology and Metabolic Diseases, Hôpital Cochin, 75014 Paris, France

11 Department of Endocrinology, Diabetes and Nutrition, CHU Grenoble-Alpes, 38043 Grenoble, France

12 Department of Endocrinology, CHRU Lille, 59037 Lille, France
13 Department of Endocrinology, Diabetes and Nutrition, CHU Bordeaux, 33604 Pessac, France

14 Department of Endocrinology, CHRU Tours, 37044 Tours, France

15 Department of Endocrinology, Diabetes and Nutrition, CHU Angers, 49933 Angers, France

16 Department of Endocrinology, Metabolic Diseases and Nutrition, Pôle Cardio-Vasculaire et Métabolique, CHU Toulouse, 31059 Toulouse, France

17 Department of Digestive and Endocrine Surgery, CHU Lyon Sud, Hospices Civils de Lyon, Université de Lyon, 69495 Pierre Bénite, France

18 Department of General and Endocrine Surgery, CHRU Lille, 59037 Lille, France

19 Department of Endocrine Surgery, CHU Nantes, 44093 Nantes, France

20 Department of Nuclear Medicine and Endocrine Oncology, Gustave Roussy Cancer Center, 94805 Villejuif, France

21 French Adrenal Cancer Network, Institut National du Cancer, 75000 Paris, France 